\title{
Correlative iPALM and Platinum Replica Electron Tomography to Highlight Single Molecules on Clathrin Endocytic Structures in 3D.
}

\author{
Kem Sochacki ${ }^{1}$, Gleb Shtengel ${ }^{2}$, Andrea Dickey ${ }^{1}$, Harald Hess ${ }^{2}$, and Justin Taraska ${ }^{1}$. \\ 1. National Heart Lung and Blood Institute, Bethesda, MD, USA. \\ 2. Janelia Research Campus, Howard Hughes Medical Institute, Ashburn, VA, USA.
}

Clathrin mediated endocytosis is a ubiquitous process used by all eukaryotic cells to internalize material. This is an integral process for neural and endocrine signaling as well as cellular homeostasis. While severe mutations to this process are lethal, minor mutations are associated with several human maladies including cancer and Alzheimer's disease [1]. There are well over 30 known proteins regulating the entire endocytic process including initiation, membrane curvature, pit size, cargo recruitment, and fission. These proteins are well biochemically characterized, but there appears to be a large amount of redundancy which has made it difficult to parse out their specific roles in endocytosis. The spatial organization of clathrin associated proteins within single clathrin structures is not well studied and may give important structural insight into how this process is regulated.

Here, we develop a correlative method that combines 3D interferometric photoactivated localization fluorescence microscopy (iPALM) and platinum replica electron microscopy (PREM) to localize clathrin associated proteins on the topography of mammalian cell membranes [2]. Platinum replicas of mammalian cell cortices viewed by transmission electron microscopy have been used for decades to survey the 3D shape of single clathrin structures across the membrane [3] (Figure 1). However, identifying proteins of interest in these images with immunogold requires large metal particles $(15 \mathrm{~nm})$ that not only label sparsely but also obstruct the image beneath. iPALM localizes fluorescently labeled proteins to a precision of better than $20 \mathrm{~nm}$ in the membrane plane (XY) and $10 \mathrm{~nm}$ in the axial (Z) plane [4]. Our iPALM/PREM correlation method allows us to correlate clathrin fluorescence with clathrin viewed with EM, to within $20 \mathrm{~nm}$ across a $20 \mu \mathrm{m}$ cell membrane. We then use this method to find the position of the clathrin adapter protein, epsin 1, with respect to the shape of clathrin structures (Figure 2). We unexpectedly find that epsin 1 is located along the edge of the clathrin structures in XY but along entire height of clathrin pits. While there are several models of how epsin functions in mammalian cells, this localization is most consistent with the recent model that epsin coordinates actin at clathrin sites.

As we continue to use this method to study other endocytic proteins, we are finding that different isoforms are localized very differently within cells and that protein localization can be very different in different mammalian cell lines. Additionally, this method is poised to answer many questions about other important processes on the plasma membrane like clathrin-independent endocytosis, exocytosis, cell adhesion, and cell motility. 


\section{References:}

[1] McMahon, Harvey T., and Emmanuel Boucrot. "Molecular mechanism and physiological functions of clathrin-mediated endocytosis." Nature reviews Molecular cell biology 12.8 (2011): 517-533.

[2] Sochacki, Kem A., et al. "Correlative super-resolution fluorescence and metal-replica transmission electron microscopy." Nature methods 11.3 (2014): 305-308.

[3] Heuser, John. "Three-dimensional visualization of coated vesicle formation in fibroblasts." The Journal of cell biology 84.3 (1980): 560-583.

[4] Shtengel, Gleb, et al. "Interferometric fluorescent super-resolution microscopy resolves 3D cellular ultrastructure." Proceedings of the National Academy of Sciences 106.9 (2009): 3125-3130.

Figure 1. Platinum Replica of U87 Glioblastoma cell line. Note the different sizes and shapes of clathrin structures marked with arrows. Scale Bar $0.5 \mu \mathrm{m}$.

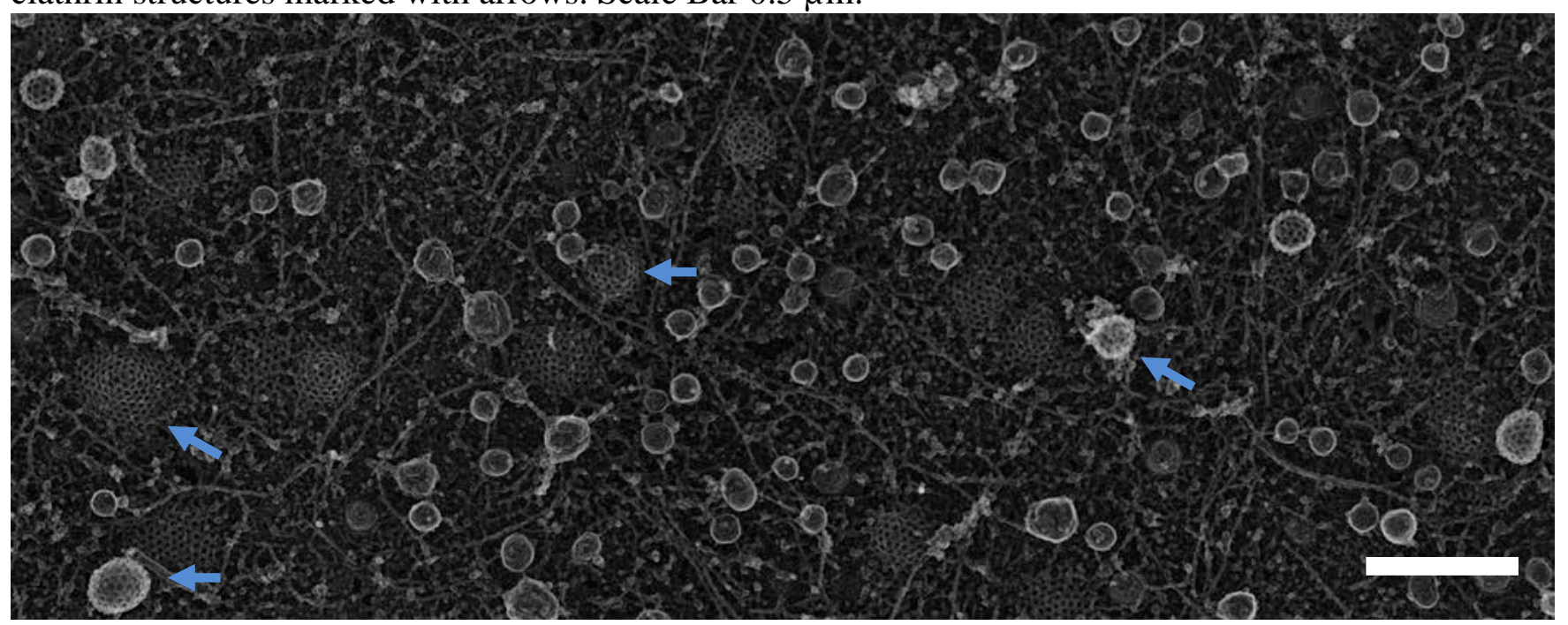

Figure 2. Correlative iPALM/PREM of Epsin1 on a clathrin coated pit. Image is $400 \mathrm{~nm} \times 400 \mathrm{~nm}$.

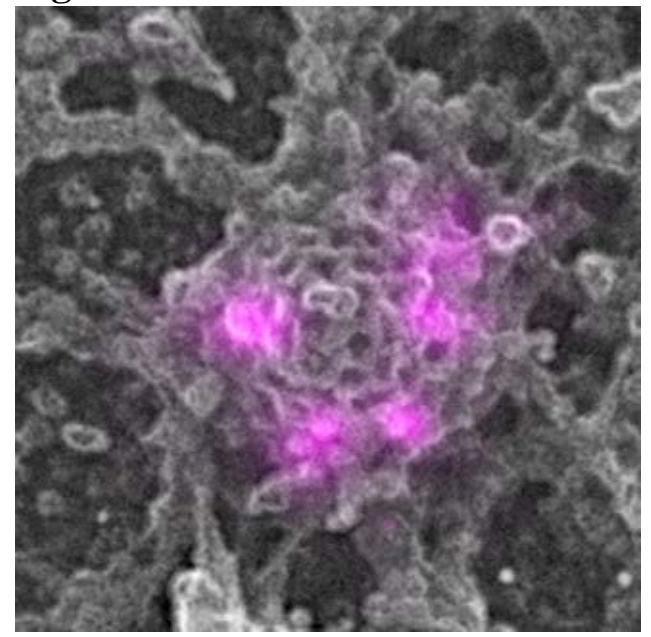

